\title{
Detection of Laser-Produced Tin Plasma Emission Lines in Atmospheric Environment by Optical Emission Spectroscopy Technique
}

\author{
Kadhim A. AADIM \\ Department of Physics, College of Science, University of Baghdad, Aljadiriya Campus, Baghdad, 00964, Iraq \\ *Corresponding author: Kadhim A. AADIM_ E-mail: kadhim_adem@scbaghdad.edu.iq
}

\begin{abstract}
A spectroscopic study on laser-produced tin plasma utilizing the optical emission spectroscopy (OES) technique is presented. Plasma is produced from a solid tin target irradiated with pulsed laser in room environment. Electron temperature is determined at different laser peak powers from the ratio of line intensities, while electron density is deduced from Saha-Boltzmann equation. A limited number of suitable tin lines are detected, and the effect of the laser peak power on the intensity of emission lines is discussed. Electron temperatures are measured in the range of $0.36 \mathrm{eV}-0.44 \mathrm{eV}$ with electron densities of the order $1017 \mathrm{~cm}^{-3}$ as the laser peak power is varied from $11 \mathrm{MW}$ to $22 \mathrm{MW}$.
\end{abstract}

Keywords: Tin plasma; laser-produced plasma; electron density; electron temperature

Citation: Kadhim A. AADIM, "Detection of Laser-Produced Tin Plasma Emission Lines in Atmospheric Environment by Optical Emission Spectroscopy Technique,” Photonic Sensors, 2017, 7(4): 289-293.

\section{Introduction}

Pulsed-laser induced plasma of solids is the subject of investigation in many fields of applied research such as laser plasma sources to x-ray lasers, inertial confinement fusion, and laboratory astrophysics [1-3]. A pulsed laser source is employed to vaporize and excite the analyte forming plasma [4]. The optical emission from the relaxation of excited species within the plasma yields information regarding the composition of the material under test [5-7].

The plasma and its characteristics (electron density, electron temperature, spatial and temporal behavior) depend on the target's thermophysical properties and laser beam parameters, such as laser pulse, temporal duration and shape, laser wavelength and energy [8-10]. Plasma descriptions start by trying to characterize the properties of the assembly of atoms, molecules, electrons, and ions rather than the individual species. If thermodynamic equilibrium exists, the plasma properties such as the description of the speed of the particles and the relative populations of energy level can be described through the concept of the temperature [11-15].

The electron temperature is an equally important plasma parameter which can be spectroscopically determined in a variety of ways: from the ratio of integrated line intensities, from the ratio of line intensity to underlying continuum, and from the shape of the continuum spectrum $[16,17]$. The diagnostic techniques employed for the determination of electron density includes plasma spectroscopy, Langmiur probe, microwave and laser interferometry, and Thomson scattering [18, 19].

Received: 18 May 2017 / Revised: 11 August 2017

(C) The Author(s) 2017. This article is published with open access at Springerlink.com

DOI: 10.1007/s13320-017-0429-x

Article type: Regular 
Spectroscopy technique is the simplest as far as instrumentation is concerned [20].

In this work, the analysis of nanosecond Nd:YAG laser-produced plasma of solid tin target in air is presented. The effect of laser peak power on the intensities of spectral lines is also reported. A study on plasma parameters, such as the electron temperature $\left(T_{e}\right)$, electron density $\left(n_{e}\right)$, and their dependencies on laser peak power, is also reported. Electron density is determined by Saha-Boltzmann equation. Electron temperature is calculated from relative intensity ratio method using Sn I $(333.06 \mathrm{~nm})$ and $\mathrm{Sn} \mathrm{I}(607.326 \mathrm{~nm})$ lines.

\section{Experiment}

The optical emission spectra of tin plasma are recorded using the experimental setup of laser-induced breakdown spectroscopy (LIBS) shown in Fig. 1.

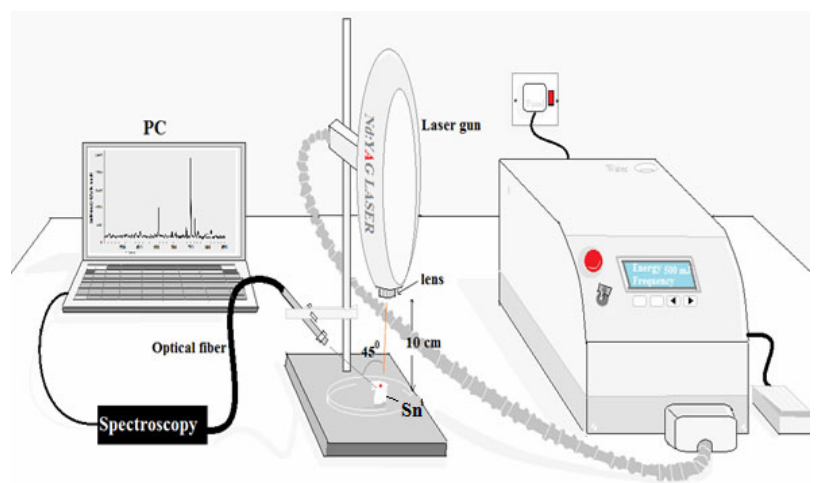

Fig. 1 Conventional LIBS system configuration used in this work.

It consists of pulsed Nd: YAG laser of $1064 \mathrm{~nm}$ wavelength, $9 \mathrm{~ns}$ duration, $1 \mathrm{~Hz}$ pulse repetition frequency, and peak power varying from $11 \mathrm{MW}$ to $22 \mathrm{MW}$. The laser beam is focused on the surface of the irradiated sample located at the focal length of a converging lens $(f=10 \mathrm{~cm})$. An optical fiber holding photodetector is adjusted at $45^{\circ}$ with beam direction at $5 \mathrm{~cm}$ distance from the sample where plasma is generated. The emission from the tin plasma plume is recorded using Ocean Optics HR 4000 CG-UV-NIR spectrum analyzer in the spectral range of $320 \mathrm{~nm}-750 \mathrm{~nm}$.

\section{Results and discussions}

The optical emission spectrum of laser-produced tin plasma in the range of $320 \mathrm{~nm}$ to $750 \mathrm{~nm}$ is shown in Fig. 2. The prominent Sn spectral lines in air ambient are Sn I (at $380.101 \mathrm{~nm}$ ), Sn I (at $452.473 \mathrm{~nm}$ ), Sn II (at $579.918 \mathrm{~nm}$ ), and Sn II (at $645.35 \mathrm{~nm}$ ). In addition, we have recorded spectral lines of low intensities such as Sn I (at $326.233 \mathrm{~nm}$ ), Sn I (at $333.06 \mathrm{~nm}$ ), Sn I (at $365.577 \mathrm{~nm}$ ), and Sn II (at $533.236 \mathrm{~nm}$ ). The transitions are identified using the spectral database of National Institute of Standards and Technology (NIST) [21].

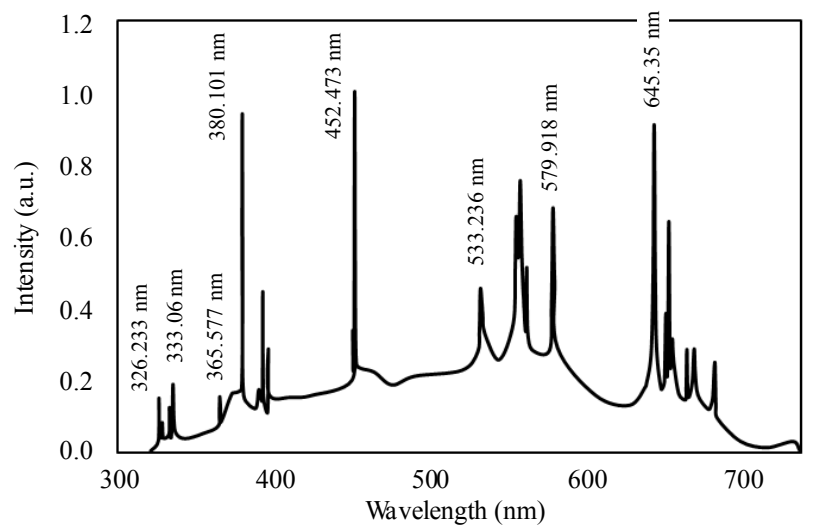

Fig. 2 Optical emission spectrum of tin plasma produced by pulsed laser of $55 \mathrm{MW}$ peak power.

The intensities of $\mathrm{Sn}$ lines at $326.233 \mathrm{~nm}$, $333.06 \mathrm{~nm}$, and $607.326 \mathrm{~nm}$ are measured at different laser peak powers. Figure 3 shows the influence of the laser peak power on the spectral line intensities. It is found that emission intensity of the spectral lines increases with an increase in the laser peak power from $22 \mathrm{MW}$ to $88 \mathrm{MW}$. This is due to the absorption of laser photon by the plasma, and at the same time the plasma is relatively transparent to the laser beam so the ablation of the target increases [22].

The increasing of target ablation produces an increase in plasma height and plasma emission. At higher values of laser peak powers, plasma shielding effect is observed, i.e., the plasma becomes opaque to the laser beam which shields the target so the lines intensities decrease. 


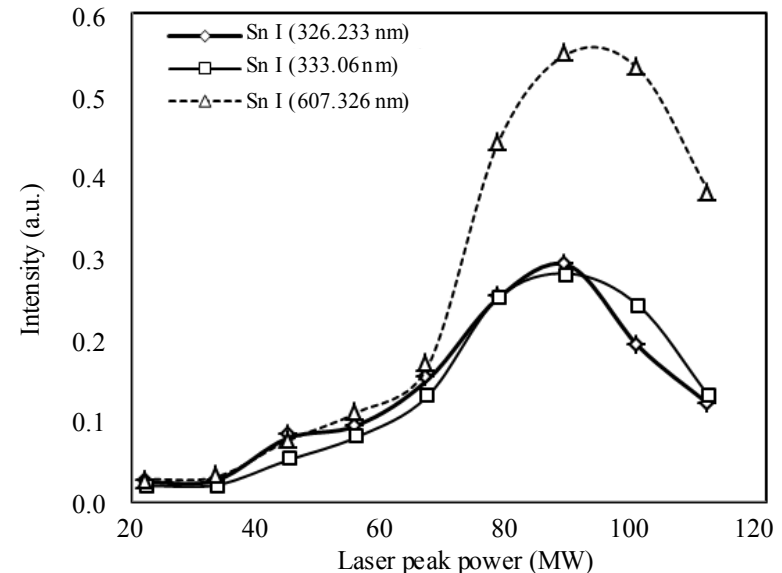

Fig. 3 Variation of emission intensity with laser peak power.

Under the assumption that the plasma is in local thermodynamic equilibrium (LTE), the lower limit of the electron density is given by McWhirter criterion [23]:

$$
n_{e} \geq 1.6 \times 10^{12} \Delta E^{3} \sqrt{T_{e}}
$$

where $n_{e}$ is the electron density, $T_{e}$ is the electron temperature, and $\Delta E$ is the difference between the energy states. The values of $n_{e}$ are obtained from Saha-Boltzmann equation as [24]

$$
n_{e}=\frac{2\left(2 \pi m_{e} k_{B} T_{e}\right)^{3 / 2}}{h^{3}} \frac{I_{m n}^{\mathrm{I}} A_{i j} g_{i}^{\mathrm{II}}}{I_{i j}^{\mathrm{II}} A_{m n} g_{m}^{\mathrm{I}}} e^{-\frac{E_{\mathrm{in}}+E_{i}^{\mathrm{II}}-E_{m}^{\mathrm{I}}}{k_{B} T_{e}}}
$$

where $m_{e}$ is the electron mass, $k_{B}$ is the Boltzmann constant, $h$ is Planck's constant, $E_{\text {ion }}$ is the ionization potential of the neutral species in its ground state, $I_{j i}$ is the intensity of the spectral line of the transition from level $j$ to $i, \lambda_{j i}$ is the wavelength, $A_{j i}$ is the transition probability, $g_{j}$ is the statistical weight, and $g_{j}$ is the energy value of higher level

Figure 4 shows the electron density of laserinduced tin plasma at different laser peak powers. It can be observed that the electron density grows as the laser peak power increases. The reason is that when a solid tin sample is irradiated by Nd: YAG laser, a collision-induced process occurs, hence free electrons in the focal volume are accelerated by the electric field of the laser and gain energy by colliding with neutral atoms. When the electrons have gained amount of energy, they can ionize atoms by collision, and this causes the electron density to grow with the laser peak power. The electron density dramatically decreases at high laser peak powers, which is due to the plasma shielding as discussed previously.

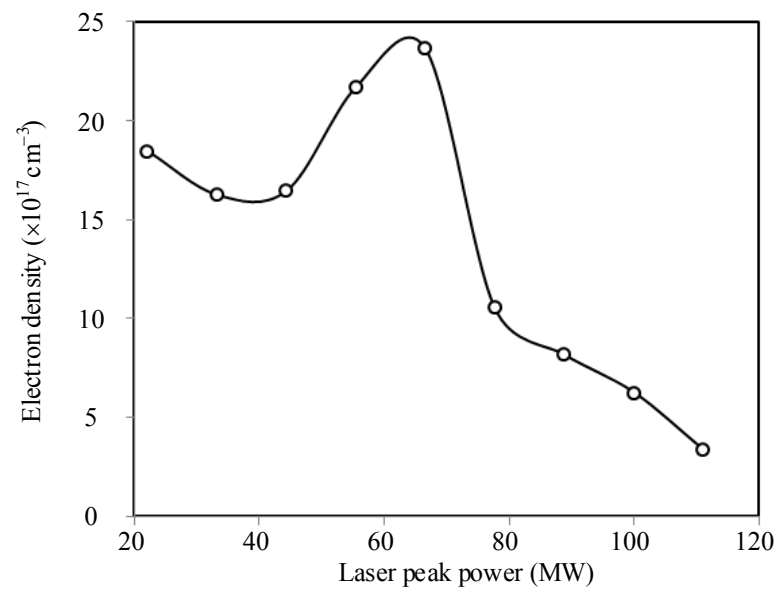

Fig. 4 Variation of electron density of laser-induced tin plasma with laser peak power.

The electron temperature $\left(T_{e}\right)$ can be calculated using the ratio of two lines of the same species of the ionization stage as

$$
T_{e}=\frac{E_{1}-E_{2}}{k_{B} \ln \left(\frac{\lambda_{2} I_{2} g_{1} A_{1}}{\lambda_{1} I_{1} g_{2} A_{2}}\right)}
$$

where $I, \lambda, g, A$, and $E$ are the total intensity, wavelength, statistical weight, absorption oscillator strength, and excitation energy of one of the lines, respectively. Primed quantities are those for the second line. These values for the two lines considered are taken from tables of the NIST [21].

The electron temperature is calculated using (3) from the ratio of the intensities of tin lines (333.06 $\mathrm{nm}$ and $607.326 \mathrm{~nm}$ ). Figure 5 shows that the electron temperature $\left(T_{e}\right)$ increases with increasing laser peak power. The electron temperature is strongly dependent on the laser peak power as the latter is the source of evaporation, atomization, and ionization of the target when focused on.

\section{Conclusions}

A tin target is irradiated by a $1064 \mathrm{~nm}$ Q-switched Nd: YAG laser to produce transient and 


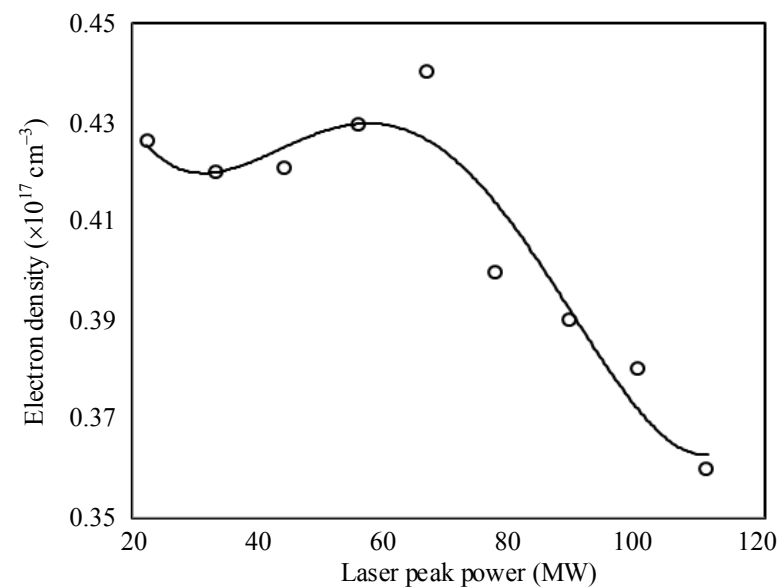

Fig. 5 Variation of electron temperature of laser-induced tin plasma with laser peak power.

elongated plasma. Measurements of electron density and temperature are carried out by the optical emission spectroscopy (OES) technique. Line intensity ratios of the successive ionization stages of the tin are used for the determination of electron temperature, and Stark broadened profile of first ionized tin species is used for the electron density measurements. The dependencies of electron density and electron temperature on different experimental parameters like distance from the target surface are studied. The electron temperature in the range of $0.36 \mathrm{eV}-0.44 \mathrm{eV}$ is obtained for the tin plasma from the Sn I line intensities while electron densities of the order of $10^{17} \mathrm{~cm}^{-3}$ are observed.

\section{Acknowledgment}

Author would like to thank Dr. Oday A. Hammadi at Al-Iraqia University for his assistance in the preparation of this manuscript.

Open Access This article is distributed under the terms of the Creative Commons Attribution 4.0 International License (http://creativecommons.org/ licenses/by/4.0/), which permits unrestricted use, distribution, and reproduction in any medium, provided you give appropriate credit to the original author(s) and the source, provide a link to the Creative Commons license, and indicate if changes were made.

\section{References}

[1] M. Autin, A. Briand, P. Mauchien, and J. M. Mermet,
"Characterization by emission spectrometry of a laser-produced plasma from a copper target in air at atmospheric pressure," Spectrochimica Acta Part B: Atomic Spectroscopy, 1993, 48(6-7): 851-862.

[2] O. A. Hammadi, M. K. Khalaf, and F. J. Kadhim, "Fabrication of UV photodetector from nickel oxide nanoparticles deposited on silicon substrate by closed-field unbalanced dual magnetron sputtering techniques," Optical and Quantum Electronics, 2015, 47(2): 1-9.

[3] M. Arab, N. Bidin, Z. H. Rizvi, S. Safie, and M. A. Alsaedi, "Comparison study of two commercial spectrometers for heavy metal analysis of laser induced breakdown spectroscopy (LIBS)," Photonic Sensors, 2014, 4(1): 63-69.

[4] J. B. Simeonsson and A. W. Miziolek, "Spectroscopic studies of laser-produced plasmas formed in $\mathrm{CO}$ and $\mathrm{CO}_{2}$ using 193, 266, 355, 532 and $1064 \mathrm{~nm}$ laser radiation," Applied Physics B, 1994, 59(1): 1-9.

[5] O. A. Hammadi, M. K. Khalafand, and F. J. Kadhim, "Silicon nitride nanostructures prepared by reactive sputtering using closed-field unbalanced dual magnetrons," Proceedings of the Institution of Mechanical Engineers Part L Journal of Materials Design \& Applications, 2017, 231(5): 479-487.

[6] D. A. Cremers and L. J. Radziemski, Handbook of laser-induced breakdown spectroscopy. West Sussex, England, Chichester: John Wiley \& Sons, Ltd., 2006: $1-275$.

[7] S. B. Zakour and H. Taleb, "Shift endpoint trace selection algorithm and wavelet analysis to detect the endpoint using optical emission spectroscopy," Photonic Sensors, 2016, 6(2): 158-168.

[8] O. A. Hamadi, "Effect of annealing on the electrical characteristics of CdO-Si heterostructure produced by plasma-induced bonding technique," Iraqi Journal of Applied Physics, 2008, 4(3): 34-37.

[9] M. A. Gigisos, S. Mar, C. Perez, and I. R. I. De, "Experimental Stark widths and shifts and transition probabilities of several Xe II lines," Physical Review E Statistical Physics Plasmas Fluids \& Related Interdisciplinary Topics, 1994, 49(2): 1575-1584.

[10] D. Zhong and Z. M. Li, "Material measurement method based on femtosecond laser plasma shock wave," Photonic Sensors, 2017, 7(1): 1-10.

[11] O. A. Hammadi, M. K. Khalaf, and F. J. Kadhim, "Fabrication and characterization of UV photodetectors based on silicon nitride nanostructures prepared by magnetron sputtering," Proceedings of the Institution of Mechanical Engineers Part $N$ Journal of Nanoengineering \& Nanosystems, 2015, 230(1): 32-36.

[12] H. R. Griem, Plasma Spectroscopy. New York: McGraw-Hill, 1964.

[13] W. L. Wiese and G. A. Martin, Wavelengths and 
transition probabilities for atoms and atomic ions, part II: Transition probabilitie. Washington, DC: National Bureau of Standards, 1980: 1-406.

[14] M. V. Allmen and A. Blatter, Laser-beam interaction with materials: physical principles and applications. Berlin: Springer-Verlag, 1995: 1-196.

[15] O. A. Hamadi, "Characteristics of CdO-Si heterostructure produced by plasma-induced bonding technique," Proceedings of the Institution of Mechanical Engineers Part L Journal of Materials Design \& Applications, 2008, 222(L1): 65-71.

[16] R. A. Ismail, O. A. Abdulrazaq, A. A. Hadi, and O. A. Hamadi, "Full characterization at $904 \mathrm{~nm}$ of large area Si p-n junction photodetectors produced by laser-induced diffusion," International Journal of Modern Physics B, 2007, 19(31): 197-201.

[17] O. A. Hammadi, "Photovoltaic properties of thermally-grown selenium-doped silicon photodiodes for infrared detection applications," Photonic Sensors, 2015, 5(2): 152-158.

[18] A. K. Yousif and O. A. Hamadi, "Plasma-induced etching of silicon surfaces," Bulgarian Journal of Physics, 2008, 35(3): 191-197.

[19] M. K. Khalaf, N. A. Al-Tememee, F. T. Ibrahim, and M. A. Hameed, "Crystalline structure and surface morphology of tin oxide films grown by DC reactive sputtering," Photonic Sensors, 2014, 4(4): 349-353.

[20] O. A. Hammadi, M. K. Khalaf, F. J. Kadhim, and B. T. Chiad, "Operation characteristics of a closed-field unbalanced dual-magnetrons plasma sputtering system," Bulgarian Journal of Physics, 2014, 41(1): 24-33.

[21] K. A. Aadim, "Optical emission spectroscopic analysis of plasma parameters in tin-copper alloy co-sputtering system," Optical and Quantum Electronics, 2016, 48(12): 545-551.

[22] O. A. Hammadi and N. E. Naji, "Electrical and spectral characterization of $\mathrm{CdS} / \mathrm{Si}$ heterojunction prepared by plasma-induced bonding," Optical and Quantum Electronics, 2016, 48(8): 1-7.

[23] A. W. Miziolek, V. Palleschi, and I. Schechter, Laser-induced breakdown spectroscopy: fundamentals and applications. Cambridge: Cambridge University Press, 2006: 1-640.

[24] K. Song, Y. L. Lee, and J. Sneddon, "Applications of laser induced breakdown spectrometry," Applied Spectroscopy Reviews, 1997, 32(3): 183-235. 